\title{
Richesse de la connaissance et champs de la pathologie
}

Observer, narrer, quantifier, discuter, rechercher, comparer, proposer, inventer, écouter, traiter, soulager, c'est toute la richesse du métier de médecin et plus particulièrement de médecin hospitalier.

L'hépatogastroentérologie est un vaste domaine médical, où se côtoient :

- la clinique médicale ;

- les connaissances biologiques et anatomopathologiques ;

- les techniques sophistiquées permettant de visualiser l'intérieur des organes du tractus digestif et de ses annexes, mais aussi de découvrir leurs structures fines grâce aux ultrasons, aux rayons $\mathrm{X}$ et aux champs magnétiques ;

- les techniques interventionnelles permettant de régler, par voie endoscopique, nombre de problèmes vitaux.

Ce champ d'action merveilleux est ouvert aux spécialistes en pathologie digestive du continent africain, qui possèdent la connaissance au même titre que leurs collègues européens et qui ont de plus en plus accès au monde complexe de la biologie moléculaire et des techniques instrumentales avancées.

L'équipement des structures hospitalières africaines se fait avec un gradient positif de l'Afrique subsaharienne vers le Maghreb et permet à un nombre croissant de spécialistes de pratiquer une médecine moderne sur un continent passionnant aux racines culturelles profondes et souvent insolites, où la pauvreté domine encore la scène. Ces difficultés existentielles d'une grande partie de la population, dans un contexte socioéconomique trop souvent perturbé, entretiennent des pathologies devenues inconnues en Occident ou de présentation très différente, car diagnostiquées à leurs prémices.

Cette richesse clinique, triste corollaire d'un manque de moyens de la population, constitue des thèmes d'études et de projets de recherche majeurs pour de jeunes spécialistes qui vont s'efforcer d'enrayer ces maladies en proposant des mesures d'amélioration de la santé publique et des protocoles thérapeutiques innovants adaptés aux moyens et au milieu, tant que faire se peut.

Le Journal africain d'hépato-gastroentérologie se veut l'organe de diffusion des travaux des spécialistes hospitaliers ou non des maladies de l'appareil digestif. Il constitue une arme de dialogue entre les médecins des pays d'Afrique francophone mais aussi entre les pays du Sud et du Nord. Il est l'organe d'expression des travaux scientifiques de qualité des services hospitaliers du continent africain orientés vers la pathologie digestive.

C'est votre journal, c'est vous qui le faites vivre!

Proposez-nous vos manuscrits, nous vous aiderons à les publier, si votre travail est innovant et soigneusement mené. Vous le méritez! 